Methods Mucosal biospy samples were taken from ulcerative colitis patients with pouchitis (chronic pouchitis $n=9$, acute pouchitis $\mathrm{n}=4$ ) and those without pouchitis $(\mathrm{n}=11)$. Epithelial cells were isolated from biopsy tissue after incubation with DTT and EDTA. Eptihelial cell expression of ZO-1, claudin 1 and claudin 2 were measured by multicolour flow cytometry. Cytokines were assessed by multiplex ELISA of biopsy supernatants. The t-test was used for statistical analysis.

Results In acute pouchitis ZO-1 was elevated compared with both chronic pouchitis and non-pouchitis $(p=0.008)$, whilst in chronic pouchtis ZO-1 expression was reduced compared with non pouchitis $(p=0.006)$. Claudin 1 expression was reduced in chronic pouchitis $(p=0.04)$, but was not significantly reduced in acute pouchitis. In acute pouchitis, claudin 2 expression was elevated ( $p \leq 0.001)$, but was not increased in chronic pouchitis. IL6 levels were elevated in chronic pouchitis compared with non pouchitis patients $(p=0.01)$. Conclusion Epithelial tight junction expression was altered in pouchitis in association with increased IL6 levels. Increased claudin 2 expression in acute, but not chronic pouchitis may represent early pathological changes in the development of pouch inflammation. In chronic inflammation the tight junction complex was deranged with reduced expression of both claudin 1 and ZO-1. Increased epithelial barrier permeability due to altered tight junction expression may be a critical mechanism in the development and perpetuation of pouch inflammation.

Disclosure of Interest None Declared.

\section{PWE-078 A PROSPECTIVE CONTROLLED PILOT STUDY OF FECAL MICROBIOTA TRANSPLANTATION FOR CHRONIC REFRACTORY POUCHITIS}

doi:10.1136/gutjnl-2013-304907.367

\begin{abstract}
1,2, J Landy, ${ }^{2} \mathrm{H} O \mathrm{Al}$-Hassi, ${ }^{2} \mathrm{E}$ R Mann, ${ }^{3} \mathrm{~S} T \mathrm{Peake},{ }^{4} \mathrm{~S}$ D McLaughlin, ${ }^{5} \mathrm{Z}$ L Perry-Woodford,

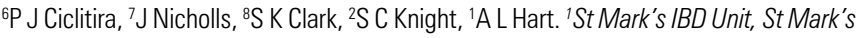
Hospital; ${ }^{2} A P R G$, Imperial College; ${ }^{3}$ BBD Unit, St Mark's Hospital, London; ${ }^{4}$ Gastroenterology, Royal Bournemouth and Christchurch Hospitals, Bournemouth; ${ }^{5}$ Pouch Care, St Mark's Hospital; ' ${ }^{6}$ Gastroenterology, St Thomas' Hospital; 'Biosurgery and Surgical Technology, Imperial college; ${ }^{8}$ Colorectal Surgery, St Mark's Hospital, London, UK
\end{abstract}

Introduction Faecal microbiota transplantation (FMT) is an effective therapy for Clostridium difficile and possibly inflammatory bowel diseases (IBD). Published data of FMT for inflammatory bowel diseases are reported in case series and case reports. To our knowledge, there are no controlled studies of FMT for IBD. We aimed to conduct a prospective study of FMT for chronic refractory pouchitis.

Methods Patients with clinically, endoscopically and histologically confirmed chronic refractory pouchitis; with a pouch disease activity index $(\mathrm{PDAI})>7$ were included. Donors were screened by clinical history and serology for HAV, HBV, HCV, HEV, Treponema, HIV, HTLV I/II and stool for M, C+S, C. difficile toxin and parasites. Fresh donor stool was collected within six hours of nasogasric administration of FMT. Stool samples were also collected from patients for analysis of coliform sensitivities before and 4 weeks after FMT. PDAI and Cleveland global quality of life score (CGQoL ) were recorded prior to FMT and four weeks after FMT.

Results Eight patients with chronic refractory pouchitis who had undergone restorative proctocolectomy for ulcerative colitis underwent FMT. Three patients had ESBL resistant coliforms on stool analysis prior toFMT. Two of these patients demonstrated a change to ciprofloxacin sensitive coliform following FMT. The mean PDAI prior to FMT was 12 . The mean CGQoL was 0.45 . At 4 weeks following FMT, no patient had achieved a clinical remission (mean PDAI 11). No improvement in CGQoL was seen (mean 0.44).

Conclusion FMT via nasogastric administration was not effective in achieving clinical remission for chronic refractory pouchitis at 4 weeks after FMT. However, in two patients with ESBL resistant coliform, ciprofloxacin sensitivity was regained following FMT and these patients have subsequently been maintained on ciprofloxacin. This suggests FMT may alter the pouch microbiota. Further molecular microbiological analysis is being undertaken to determine the effect FMT had on these patients' microbiota. In addition, further studies of FMT are required to assess the effect of different methods of FMT that may be more efficacious for this group of patients.

Disclosure of Interest None Declared.

\section{PWE-079 LONGITUDINAL ASSESSMENT OF EPITHELIAL AND IMMUNE CELL CHANGES FOLLOWING ILEOSTOMY CLOSURE IN PATIENTS WITH ULCERATIVE COLITIS}

doi:10.1136/gutjinl-2013-304907.368

1.2, J Landy, ${ }^{2} \mathrm{H} O \mathrm{Al}$-Hassi, ${ }^{2} \mathrm{E}$ R Mann, ${ }^{3} \mathrm{~S}$ T Peake, ${ }^{4 \mathrm{P}} \mathrm{J}$ Ciclitira, ${ }^{5} \mathrm{~J}$ Nicholls, ${ }^{6} \mathrm{~S}$ K Clark, ${ }^{2} S$ C Knight, ' 'A L Hart. 'St Mark's IBD Unit, St Mark's Hospital; ${ }^{2} A P R G$, Imperial College; ${ }^{3}$ IBD Unit, St Mark's Hospital; " Gastroenterology, St Thomas' Hospital; ${ }^{5}$ Biosurgery and Surgical Technology, Imperial college; ${ }^{6}$ Colorectal Surgery, St Mark's Hospital, London, UK

Introduction The interactions between microbiota, epithelial barrier and innate immune responses are important in the pathogenesis of IBD. The ileo-anal pouch offers a unique opportunity to study these inter-relationships before the onset of disease. There are few data regarding tight junction expression or dendritic cell (DC) characteristics following restorative proctocolectomy (RPC). We aimed to assess the relationship between changes in epithelial tight junction expression, dendritic cell phenotype and mucosal cytokine production over the first year following RPC for ulcerative colitis (UC). Methods Mucosal biopsy samples were taken from the same UC patients undergoing RPC, from the ileostomy afferent loop, the pouch pre-ileostomy closure (P0) and the pouch 6 and 12 months post-ileostomy closure $(n=5)$. Eptihelial cells expression of zona occludens (ZO)-1, claudin 1 and claudin 2 and DC expression of TLR 2 and 4, CCR9, $\beta 7$ and CD40 were measured by multicolour flow cytometry. Cytokines were assessed by multiplex ELISA of biopsy supernatants. The paired t-test was used for statistical analysis.

Results Epithelial expression of claudin 2 was increased $(p=0.04)$ at 6 months and remained elevated at 12 months. No changes were seen in ZO-1 or claudin 1 expression. There was a significant increase in $\beta 7$ expression on lamina propria DC $(p=0.02)$, but no differences in DC TLR or CD40 expresssion were seen at 6 months. DC expression of $\beta 7$ was further elevated $(p=0.005)$ as well as significantly increased TLR 4 and CD40 expression $(p=0.04)$. No cytokines were found to be elevated at 6 months, but at 12 months there was a trend towards increased IL6 $(p=0.05)$.

Conclusion In patients with UC, altered tight junction expression with increased epithelial expression of the "pore-forming" tight junction claudin 2 was an early event after ileostomy closure that preceded the onset of mucosal inflammation. In parallel, more lamina propria DC expressed gut homing markers possibly in response to increased exposure to the changing microbial signals and a more permeable epithelial barrier. These changes in parallel may lead to increased microbial stimulation of DC with increased TLR and costimulatory molecule expression that could predispose to the development of inflammation.

Disclosure of Interest J. Landy Grant/Research Support from: The Broad Foundation, H. Al-Hassi: None Declared, E. Mann: None Declared, S. Peake: None Declared, P. Ciclitira: None Declared, J. Nicholls: None Declared, S. Clark: None Declared, S. Knight: None Declared, A. Hart: None Declared

\section{PWE-080 PREVALENCE OF FAECAL INCONTINENCE IN ADULTS} WITH INFLAMMATORY BOWEL DISEASE

doi:10.1136/gutjnl-2013-304907.369 
1."J Duncan, ' $\mathrm{G}$ Sebepos-Rogers, ${ }^{2} \mathrm{O}$ Poole-Wilson, ${ }^{3} \mathrm{C}$ To, ${ }^{4} \mathrm{~J}$ B Canavan, ${ }^{1} \mathrm{M}$ Ward, ${ }^{1} \mathrm{~V}$ Kariyawasam, 'R Goel, ' ${ }^{1} \mathrm{~K}$ Patel, 'A Stanton, 'M Sastrillo, 'S Anderson, ' $\mathrm{K}$ Taylor, ' $\mathrm{J}$ Sanderson, 'P Irving. 'Gastroenterology, Guy's \& St Thomas' NHS Foundation Trust; ${ }^{2}$ South London Healthcare Trust, London, UK; ${ }^{3}$ Australian National University, Canberra, Australia; ${ }^{4}$ Kings College London, London, UK

Introduction The prevalence of faecal incontinence (FI) in people with inflammatory bowel disease (IBD) has not been fully explored. FI is not only associated with social stigma but also with decreased quality of life. In the general population prevalence is estimated at between 1-10\%. Awareness of the prevalence of FI in IBD is important to aid management strategies and resource allocation.

Methods Aim: To investigate the prevalence of FI in adults with IBD in a tertiary care setting. Methods: We performed a cross sectional questionnaire survey of 380 adults attending IBD outpatients at Guy's \& St.Thomas' Hospitals. Patient surveys were: the validated International Consultation on Incontinence - Bowels (ICIOB) questionnaire, detailing frequency and severity of bowel pattern, control and quality of life; and the non-validated Bowel Leakage questionnaire, detailing any prior interventions by health care professionals. Demographics of age, gender, diagnosis, Montreal classification, St Mark's Continence Score and disease activity were also recorded. Data was entered into a database and analysed using a SPSS statistical package.

Results Median age was 38 years (IOR 31-50) and 180/380 $(47 \%)$ were female. The mean duration of IBD diagnosis was 8.7 years (3.4-15.1). 151/380 (40\%) had UC vs 229/380 (60\%) CD. Overall, $255 / 380(67 \%)$ reported FI as defined by any episode of uncontrolled bowel opening in the preceding three months, while $343 / 380(90 \%)$ reported anal incontinence of flatus or faeces. Incontinence was strongly associated with disease activity, occurring during disease flares in $57 \%$ of people. However, $37 \%$ experienced incontinence both during relapse and remission, whilst only $5 \%$ experienced incontinence uniquely when in remission. The ICIO-B control score was associated with current disease activity in CD $(r=0.29, p<0.0001)$ but not in UC. There was no significant difference in FI prevalence between patients with Crohn's Disease (CD) or Ulcerative Colitis (UC), (66\% vs $68 \%$, $\mathrm{p}=0.74)$

Conclusion Faecal incontinence in IBD increases in proportion to disease activity. Given the availability of specialist FI interventions and support, we recommend that sensitive questioning regarding FI should be part of routine disease surveillance in the outpatient setting to cater for this unmet need.

Disclosure of Interest None Declared.

\section{PWE-081 THIOGUANINE REMAINS AN ACCEPTABLE ALTERNATIVE IMMUNOSUPPRESSANT IN IBD}

doi:10.1136/gutjnl-2013-304907.370

1." K V Patel, 'R M Goel, ' $\mathrm{V}$ C Kariyawasam, 1 P A Blaker, 'M G Ward, ${ }^{2}$ S Mashari, ${ }^{2}$ A Clark, 'M Sastrillo, 'J Duncan, 'S H Anderson, 'P M Irving, 'J D Sanderson. 'Gastroenterology; 2Pharmacy, Guy's and St Thomas' NHS Foundation Trust, London, UK

Introduction A significant proportion of inflammatory bowel disease (IBD) patients do not tolerate conventional thiopurines (azathioprine (AZA) and mercaptopurine (MP)). Thioguanine (TG) remains an alternative immunomodulator (IM) and has been in regular use in the IBD clinic at Guy's \& St. Thomas' for more than 10 years. Concerns remain regarding nodular regenerative hyperplasia (NRH) in long-term use.

Methods We aimed to describe the indications, efficacy, monitoring and sequelae of TG use in IBD patients. Patients prescribed TG for IBD from 2008-2012, subsequent to a previously published series, were reviewed.
Results 37 patients were identified (15 male, mean age 43 years). 29 patients had Crohn's disease with 2 having co-existing orofacial granulomatosis, 6 had ulcerative colitis and 1 had IBD-unclassified. All had previous exposure to AZA and/or MP. The indications for TG were thiopurine intolerance in 23 and thiopurine induced pancreatitis in 13 patients.

Median duration of exposure was 11 months (IOR: 3-30). Maintenance doses were $20 \mathrm{mg}$ or $40 \mathrm{mg}$. 20 patients (54\%) were in clinical remission at the end of follow-up period, and $16(41 \%)$ of these were steroid free. Mean TGN levels were $1177 \mathrm{pmol} / 8 \times 10^{8} \mathrm{RBC}$ (range $\left.70-3182 \mathrm{pmol} / 8 \times 10^{8}\right)$. Differences in TGN concentrations in clinical responders and non-responders were not statistically significant.

Treatment was withdrawn in 7 patients. Withdrawal was for adverse events in 3 patients, which resolved on TG withdrawal (neutropenic sepsis, pancytopenia and AST rise) and treatment failure in further 3 patients. TG was ceased in another after the diagnosis of metastatic breast cancer whilst receiving Adalimumab and concomitant TG for 12 months. 7 patients discontinued TG because of side effects occurring within 2 months of initiation.

11 patients had long-term exposure (> 18 months). Screening with liver biopsy and/or MRI-Liver was performed in 9 patients. All patients were screened with regular platelet counts and liver profile. None were diagnosed with NRH during follow-up.

Conclusion TG is a viable second-line IM and appears to be effective with an acceptable safety profile. Side effects are uncommon, occur early and cease upon TG withdrawal. Chief indications are previous failure of immunosuppressant therapy or thiopurine-associated pancreatitis. There were no cases of TG-associated pancreatitis. Concerns regarding hepatotoxicity and NRH were not manifest in this small series of patients in concordance with results from similar recent European series. Dedicated liver MRI screening along with quarterly liver profile and platelet counts to monitor for NRH should be offered to patients on long term therapy.

Disclosure of Interest None Declared.

\section{PWE-082 W/THDRAWN BY AUTHOR}

\section{PWE-083 THE DEVELOPMENT OF A STRATIFIED MODEL OF FOLLOW UP CARE FOR ADULT PATIENTS WITH INFLAMMATORY BOWEL DISEASE}

doi:10.1136/gutjnl-2013-304907.371

1."K Kemp, ${ }^{2} \mathrm{~J}$ Griffiths, ${ }^{3} \mathrm{~S}$ Campbell, ${ }^{2} \mathrm{~K}$ Lovell. 'School of Nursing/Gastroenterology, University of Manchester/Manchester Royal Infirm, Manchester; ${ }^{2}$ School of Nursing, University of Manchester, Manchester; ${ }^{3}$ Gastroenterology, Manchester Royal Infirmary, Manchester, UK

Introduction There is concern in the UK that services for pts with long term conditions are not orgnised to promote independence with silo working in primary and secondary care with reactive services. These may be brought together formally through the development of model of care. Utilization of current out-pt spaces to regularly review stable pts is inappropriate and is challenged by commissioners. The question remains as to what models of follow up (FU) are we able to offer pts which are acceptable and feasible. The aim of this study was to develop an integrated, acceptable, modern model of FU care for pts with IBD.

Methods Using the MRC Framework for complex interventions, 24 IBDs (18 pts had CD, $6 \mathrm{UC}$, age range 27-72 years, disease duration range $2-40 \mathrm{yr}$ ), 20 purposively selected GPs from NW England, and 3 IBD Nurses (specialist, advanced practitioner, consultant nurse) were interviewed. Participants were asked about the role of FU in IBD, experience of FU patterns, service delivery, other models of FU. Thematic analysis was undertaken using NVivo 9.0. Analysis of 3 groups of interviews were synthesised by a Cons Gastroenterologist, patient, GP, IBD Nurse, to develop the model of FU care. 